\title{
Investigation of Unsteady/Quasi-Steady Scramjet Behavior using High-Speed Visualization Techniques
}

\author{
Stuart J. Laurence ${ }^{1}$, Hiroshi Ozawa ${ }^{2}$, Damien Lieber ${ }^{3}$, Jan Martinez Schramm ${ }^{2}$, and Klaus Hannemann ${ }^{4}$ \\ German Aerospace Center (DLR), Göttingen, D 37073, Germany
}

\begin{abstract}
Experiments are carried out in the HEG (High Enthalpy Shock Tunnel Göttingen) windtunnel to obtain detailed measurements on the HyShot II scramjet configuration at equivalence ratios close to the incipient choking point at simulated 28-km altitude flight conditions. Diagnostic techniques include time-resolved pressure measurements and simultaneous high-speed schlieren and $\mathrm{OH}^{*}$ chemiluminescence imaging. Similar to previous experiments at higher equivalence ratios, the onset of choking is signaled by the formation of an unsteady shock train that initially propagates up the duct. The shock motion then slows, however, and a quasi-steady topology develops with the shock lodged at a position that depends strongly on the equivalence ratio (the position lying further upstream for higher equivalence ratios). This topology persists until the conclusion of the steady test time. Based on these measurements, a value for the critical choking equivalence ratio, i.e., that at which the shock train first appears, in the range of 0.38-0.39 is determined. High-speed temperature sensitive paint measurements are also carried out on the model intake ramp to provide global information on the boundary-layer transition behavior.
\end{abstract}

\section{Introduction}

U NSTART in scramjet engines is a process whereby the original inlet shock system is displaced upstream and "disgorged" out of the intake ${ }^{1}$. This phenomenon is highly undesirable: the resulting flow spillage reduces engine performance, and even more importantly, the violent, unsteady oscillations produced by the detached shock can lead to destruction of the vehicle. The failure of the second X-51 flight experiment, for example, was attributed to such an unstart event ${ }^{2}$. One possible cause of unstart is the development of abnormal operating conditions in the combustion chamber, resulting in pressure disturbances that propagate upstream and affect the inlet flow. Such abnormalities in the operating conditions invariably involve one of two phenomena (or a combination thereof): (1) excessive heat release driving the flow to sonic conditions, i.e., thermally choking; (2) the adverse pressure gradient produced by combustion causing the boundary layer to separate, resulting in the formation of an oblique shock train which can propagate upstream. Experimental investigations of the processes associated with combustor-originating unstart often replace the combustion-induced pressure rise with mechanical throttling ${ }^{3-5}$, allowing the use of cold flows but limiting the information that can be gathered regarding the source of the choking behavior. Studies involving combusting flows ${ }^{6,7}$, on the other hand, have typically been hampered by limited diagnostics.

In earlier work ${ }^{8}$, we carried out experiments on a scale reproduction of the HyShot II configuration at simulated $28-\mathrm{km}$ altitude flight conditions in the HEG reflected-shock wind tunnel. HyShot II was a flight experiment conducted by The University of Queensland in Australia ${ }^{9}$, designed to provide benchmark data on supersonic combustion for a flight Mach number of approximately $M=8$. In our previous experiments, we employed timeresolved surface pressure measurements, and high-speed schlieren and $\mathrm{OH}^{*}$ imaging (non-simultaneously) to gain insight into the transient phenomena associated with the choking of the HyShot II combustor at high equivalenceratio conditions. The onset of unstart was signaled by the formation of an unsteady shock train that propagated up the duct at a speed that increased with increasing equivalence ratio (approximately $90 \mathrm{~m} / \mathrm{s}$ for ER=0.7). The mechanism responsible for the development of this shock train was identified as thermal choking, based on a lack of evidence for large-scale boundary separation during the formation stage. Further, computational fluid dynamics (CFD) simulations ${ }^{10}$, showed that this choking was not occurring in a global sense over the entire combustor cross-

\footnotetext{
${ }^{1}$ Research Staff, Institute of Aerodynamics and Flow Technology, Spacecraft Department, AIAA Member.

${ }^{2}$ Research Staff, Institute of Aerodynamics and Flow Technology, Spacecraft Department.

${ }^{3}$ Student Intern, Institute of Aerodynamics and Flow Technology.

${ }^{4}$ Head Spacecraft Department, Institute of Aerodynamics and Flow Technology, AIAA Senior Member.
} 
section, but rather was limited to the region of main heat release near the shear layer between the injected hydrogen and incoming air flow. Despite its localized nature, this "local" thermal choking dictates the global behavior of the combustor. In $\mathrm{ER}=0.7$ experiments, following the initial phase of shock propagation up the combustion chamber, a quasi-steady flow topology developed with the leading shock lodged just downstream of the injectors. This topology persisted until the conclusion of the steady test time.

In the present investigation, an identical experimental configuration is employed, but emphasis is placed on lower equivalence ratios closer to the value at which this unsteady choking behavior was first expected to appear. The aims are both to identify this critical value of the equivalence ratio and to improve our understanding of the combustion and fluid dynamical phenomena in this operating regime. The use of improved diagnostic techniques, in particular the availability of a second high-speed camera, enabled a larger set of information to be obtained from each shot, which in turn allowed a range of equivalence ratios to be explored. Further high-speed visualizations consisting of temperature sensitive paint measurements on the intake ramp provided a global picture of the twodimensional transition behavior of the ramp boundary layer.

\section{Experimental facility and model}

All experiments were carried out in the HEG (High Enthalpy shock tunnel Göttingen) facility of the German Aerospace Center. The HEG is a reflected-shock tunnel, capable of producing flows over a wide range of stagnation pressures and total enthalpies. A schematic of the facility is shown in Figure 1. Further information on the operating principles and conditions achievable in HEG is provided in refs. $11 \& 12$. Briefly, to initiate a test run, compressed air is used to accelerate a free-piston down the compression tube, which is filled typically with a mixture of helium and argon. A primary diaphragm separates the compression tube from the shock tube, containing the test gas: in this case, air. When the pressure in front of the piston becomes sufficiently high, the primary diaphragm bursts, sending a strong shock wave down the shock tube. This shock reflects from the far end of the shock tube, bursting the secondary (mylar) diaphragm, which separates the tunnel nozzle and test section, initially under vacuum, from the shock tube. The reflected shock also decelerates the test gas in the shock tube to stagnation conditions, forming the reservoir for the subsequent expansion through the hypersonic nozzle and into the test section. Steady test flow conditions persist typically for several milliseconds; the test time is terminated either by the arrival of the expansion wave from the primary diaphragm burst, or by contamination of the test flow by the driver (compression tube) gas.
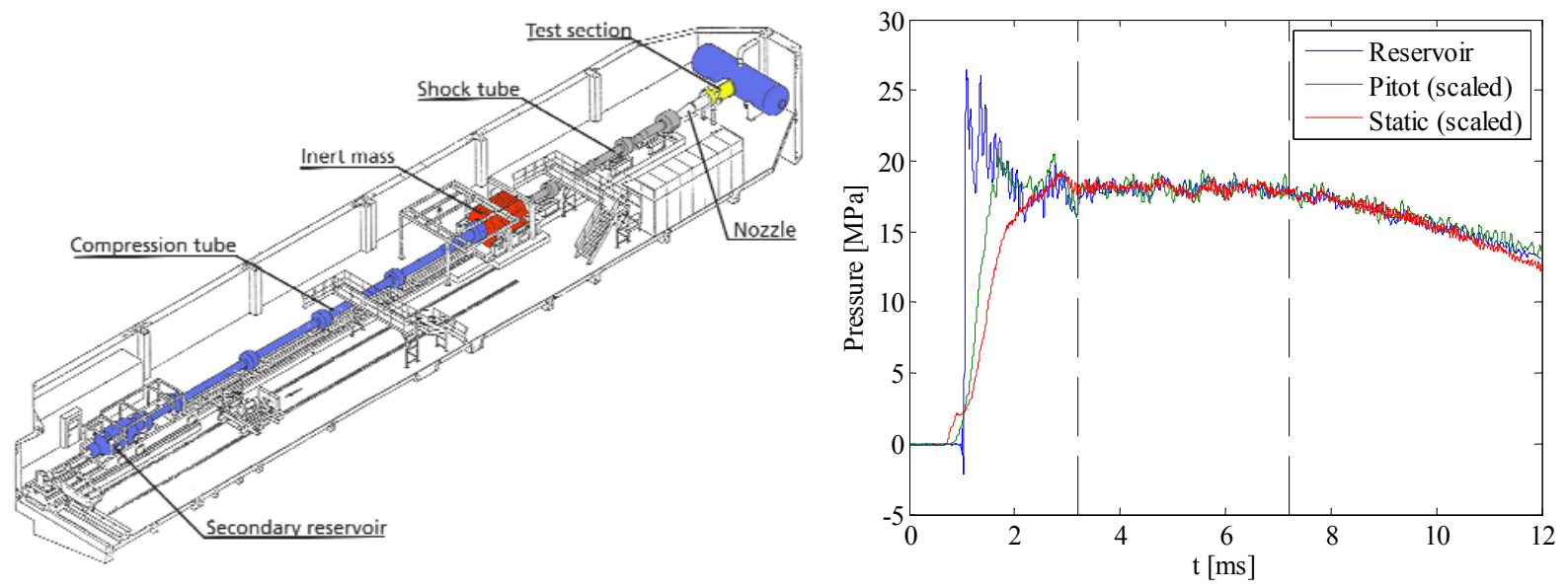

Figure 1: (Left) Schematic of the HEG reflected shock-tunnel facility; (right) reservoir, scaled Pitot, and scaled static pressures for the HEG condition used in the present experiments. The dashed vertical lines indicate the steady test time.

For all tests in the current study, a modified version of HEG Condition XIII, designed to simulate the flight conditions of the HyShot II vehicle at $28 \mathrm{~km}$ altitude, was employed. The mean reservoir pressure and enthalpy for the modified condition over the test sequence were $18.4 \mathrm{MPa}$ and $3.2 \mathrm{MJ} / \mathrm{kg}$, respectively; a typical reservoir pressure trace is shown in the right plot of figure 1, together with scaled free-stream Pitot and static pressure measurements. The modifications to the test condition were made in order to address the slight undertailoring present in earlier experiments. The improvement in the steadiness of the flow and the duration of the test time can be seen by comparing the right plot in Figure 1 with the corresponding trace in figure 2 of ref. 8 . The steady test time, shown in vertical dashed lines, is now of the order of $4 \mathrm{~ms}$ (here from 3.2 to $7.2 \mathrm{~ms}$ ), and the variability in the 
measured pressures during this time is reduced. The free-stream properties corresponding to earlier Condition XIII reservoir conditions have been calculated computationally and compared with extensive calibration measurements; applying scaling laws to these, we arrive at free-stream conditions in the present experiments of $\mathrm{M}=7.4, \mathrm{~T}=260 \mathrm{~K}$, and $\rho=0.027 \mathrm{~kg} / \mathrm{m}^{3}$. It has been shown that comparing the static pressure development to that of the reservoir and Pitot pressures gives a good indication of the arrival of the driver-gas contamination ${ }^{12}$ : the Pitot pressure is typically unaffected by driver gas contamination and follows the reservoir pressure regardless, but the static pressure drops more quickly once driver gas is present in the free stream. In Figure 1, we see that the static pressure development follows that of the reservoir and Pitot pressures until at least $10 \mathrm{~ms}$, indicating that there is no significant contamination during this time. The steady test time is instead terminated by the arrival of expansion waves from the primary-diaphragm burst.

The HyShot II model used in the present experiments was identical to that employed in the earlier study described in ref. 8. A schematic of the model in the HEG test section and a sketch of the flow path are shown in Figure 2 (note that the geometrical convention adopted throughout this paper has the combustion chamber flipped vertically relative to the physical configuration). The model geometry consists of a simple two-dimensional configuration designed to provide reference supersonic combustion data, rather than to produce thrust at the tested conditions. The intake is an $18^{\circ} \mathrm{ramp}, 196 \mathrm{~mm}$ wide and $350 \mathrm{~mm}$ long, with side walls to ensure the twodimensionality of the flow entering the combustion chamber. The combustion chamber consists of a constant-area duct of length $300 \mathrm{~mm}$, followed by a simple expansion formed by two straight-edged thrust surfaces. The constantarea combustion chamber is $9.8 \mathrm{~mm}$ high by $75 \mathrm{~mm}$ wide. A boundary-layer bleed channel is employed to prevent the intake boundary-layer from entering the combustion chamber; this also serves to swallow the shock generated at the leading edge of the cowl. Windows were installed in the combustion chamber side-walls in order to allow schlieren and $\mathrm{OH}^{*}$ visualizations to be obtained. For $\mathrm{OH}^{*}$ visualization, quartz windows were employed on both the model and test section to allow transmittance of the relevant wavelength $(\sim 310 \mathrm{~nm})$. For all experiments, the model was placed at an angle-of-attack of $3.6^{\circ}$ (i.e., so that the ramp angle was $21.6^{\circ}$ to the free stream).

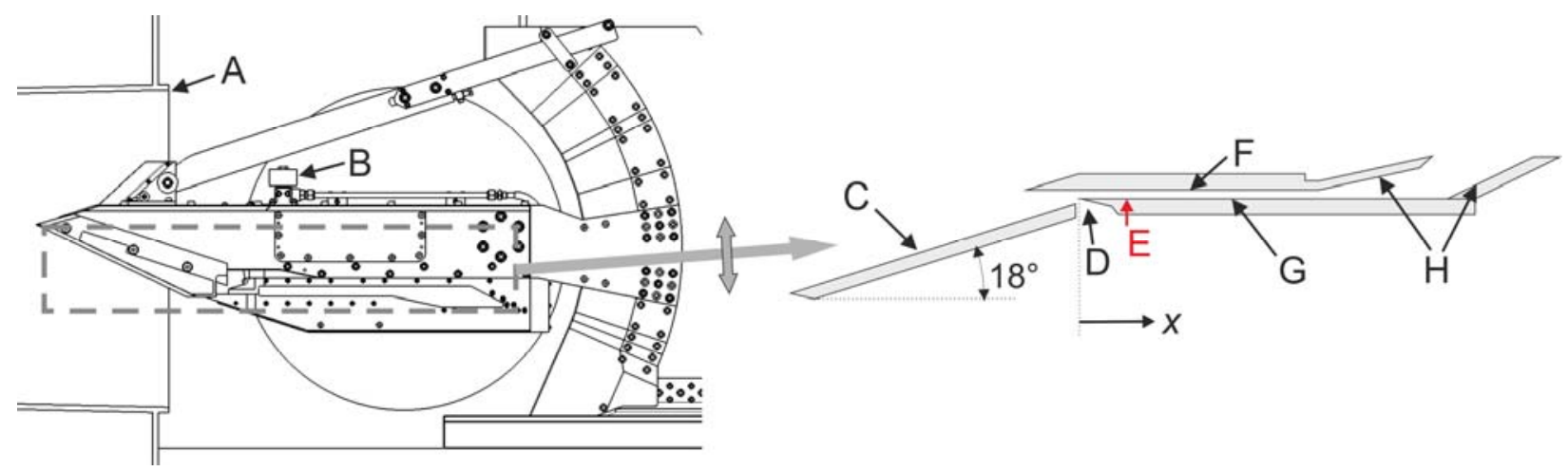

Figure 2: (Left) Schematic of the HyShot II model inside the HEG test section and (right) sketch of the model flowpath (upside down relative to the left schematic): (A) HEG nozzle; (B) $\mathrm{H}_{2}$ injection valve; (C) intake ramp; (D) boundary-layer bleed channel/shock trap; (E) injection location; (F) cowl-side wall; (G) injectorside wall; (H) expansion surfaces.

Hydrogen fuel was injected in the wall-normal direction through four evenly spaced holes on the ramp side of the combustion chamber, at a distance $58 \mathrm{~mm}$ downstream from the leading edge of this wall (i.e., from the beginning of the constant area section). The porthole injectors were each of radius $1 \mathrm{~mm}$, providing a total injection area of $12.6 \mathrm{~mm}^{2}$. The hydrogen was provided by a Ludwieg tube, capable of providing an approximately constant pulse of fuel for $50 \mathrm{~ms}$; fill pressures here were in the range of 7 to 10 bars. To ensure the injection was fully established by the arrival of the test flow, injection was triggered in each run approximately $25 \mathrm{~ms}$ before the test time by the pressure rise in the compression tube.

\section{Temperature Sensitive Paint Measurements on Intake Ramp}

In order to gain an understanding of the global transition behavior on the intake ramp, standard thermocouple measurements were supplemented by the use of temperature sensitive paint (TSP). Temperature sensitive paints have been widely used in longer duration hypersonic facilities for global heat flux measurement and the identification of transition locations ${ }^{13-15}$; their ability to provide full-field measurements makes them an attractive 
and economical alternative to discrete sensors such as thermocouples and thin-film gages. Their use in millisecondduration facilities such as HEG, however, has been more constrained by conflicting requirements of a fast response time (the response time being proportional to the square of the paint thickness, this requirement favors a thin TSP layer) and high luminous intensity to overcome the self-luminosity produced by the facility (making a thicker TSP layer advantageous). Nevertheless, with the continuing development of high intensity LEDs, providing readily available and convenient light sources, together with advances in high-speed camera technology, time-resolved TSP techniques are becoming a realistic option for heat-flux measurements and transition-location determination even in facilities such as HEG.

\section{A. Technique Description}

For the present measurements, the intake ramp was coated with a $\sim 0.5 \mathrm{~mm}$-thick polyurethane insulating layer, to which a $\sim 10 \mu \mathrm{m}$ coating of TSP was added. A UV-sensitive luminophore was chosen together with a nylon polymer and ethanol as binder and solvent, respectively. A spray gun was used to apply both the insulating layer and the TSP solution. The TSP was excited with two UV-LEDs, with peak emission at $380 \mathrm{~nm}$. Images were recorded with a Phantom v1210 high speed camera at a frame rate of 40kfps, a resolution of 512x480 pixels, and an exposure time of $24 \mu \mathrm{s}$. To exclude non-TSP related emission, a bandpass filter of $40 \mathrm{~nm}$ width, centered at $435 \mathrm{~nm}$, was placed in front of the camera lens.

The image intensity profiles recorded during experiments were converted into temperature data using a predetermined calibration. Similar to other authors, a polynomial approximation was used to the expected Arrhenius profile. It should be noted the calibration facility was only able to produce temperatures as high as $323 \mathrm{~K}$; above this value, the polynomial approximation can no longer be considered valid. Despite the use of the bandpass filter, facility luminosity was found to be a problem during the flow start-up period. To prevent the erroneous temperatures recorded during this period from influencing the heat-flux values during the subsequent steady test time, the affected sections of the temperature profiles were manually replaced with the fitted parabolic forms expected if the heat flux were constant over this time. The time-resolved temperature profiles were then converted into heat flux curves using the numerical routine proposed in ref. 16. Considering the thickness of the TSP layer, the use of a-priori thermal properties for the insulating layer was not expected to give reliable results for the heat-flux determination (see, for example, ref. 17); thus, a row of four thermocouples was installed on the ramp centerline in order to provide an insitu calibration.

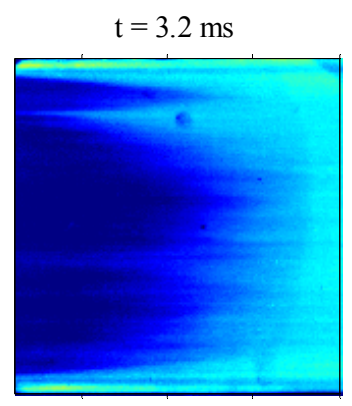

$\begin{array}{llll}150 & 200 & 250 & 300\end{array}$

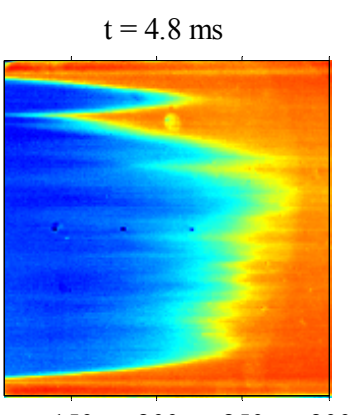

150

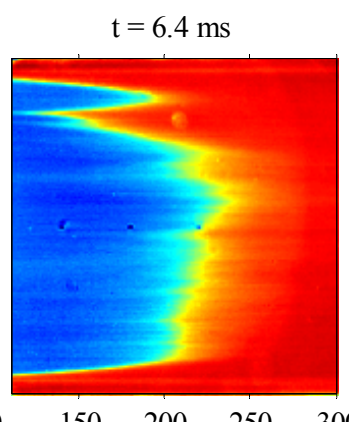

$\begin{array}{llll}150 & 200 & 250 & 300\end{array}$

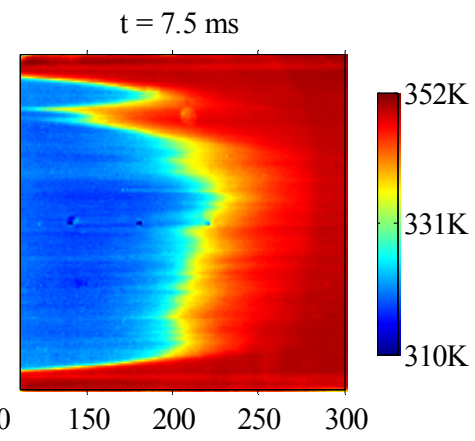

Distance from leading edge $[\mathrm{mm}]$

Figure 3: Instantaneous temperature distributions on the intake ramp at four different times in an HEG experiment

\section{B. Results}

In figure 3 we show instantaneous temperature distributions on the intake at four time instants. The three rear thermocouples are visible in these images. The derived heat-flux distribution during the steady test time is shown in the right part of figure 4; the quantitative heat-flux profile on the central line along which the thermocouples lie is compared with the thermocouple measurements in the left part of this figure. Also shown here are the results of thermocouple measurements on a steel ramp to which no TSP was applied (but which was otherwise identical). The two-dimensional transition "line" can be clearly identified from the sharply rising in heat-fluxes in the figure 4 distribution, and lies at the approximate streamwise location of the rearmost thermocouple. The transition location is roughly constant over much of the ramp; the notable double-strake present in the upper part of the image was determined to have resulted from two small (unintentional) roughness elements on the TSP surface. The shocks 
generated by the side-walls of the intake are also clearly causing almost immediate transition downstream. In the temperature distributions of figure 3, the temperature in the transitional region is seen to exceed the maximum calibration temperature; thus, the quantitative temperature and heat-flux values here cannot be considered reliable. Nevertheless, the temperatures in the laminar region remain within the valid bounds and the global transition location can be clearly identified, illustrating the usefulness of TSP measurements in the current configuration.

In figure 4, the most-upstream part of the TSP-measured heat-flux curve has been matched to the thermocouple value, so it is not surprising that they show good agreement. The error bars in both cases show the time-variation in the numerically determined heat flux during the test time; for the TSP measurements, the errors in only the first and last points are plotted to avoid obscuring the other data. The expected decrease in heat-flux in the laminar flow region can be observed in both the TSP and thermocouple profiles, followed by a steep rise indicating the onset of transition. Overall, the agreement between the TSP and (limited) thermocouple measurements is very satisfactory, providing some degree of confidence in the TSP-derived values, at least within the streamwise range of the thermocouple measurements. As already noted, the TSP values further downstream cannot be considered reliable because of the lack of an appropriate calibration; the plateau in the TSP-measured heat flux should thus be treated with caution and is most likely unphysical. Comparing the TSP-coated and steel ramp results, a small ( 25mm) delay in transition is observed for the former, and this was found to be consistent over a number of other experiments. This is perhaps due to the effective increase in leading-edge radius caused by the TSP base layer delaying transition slightly.
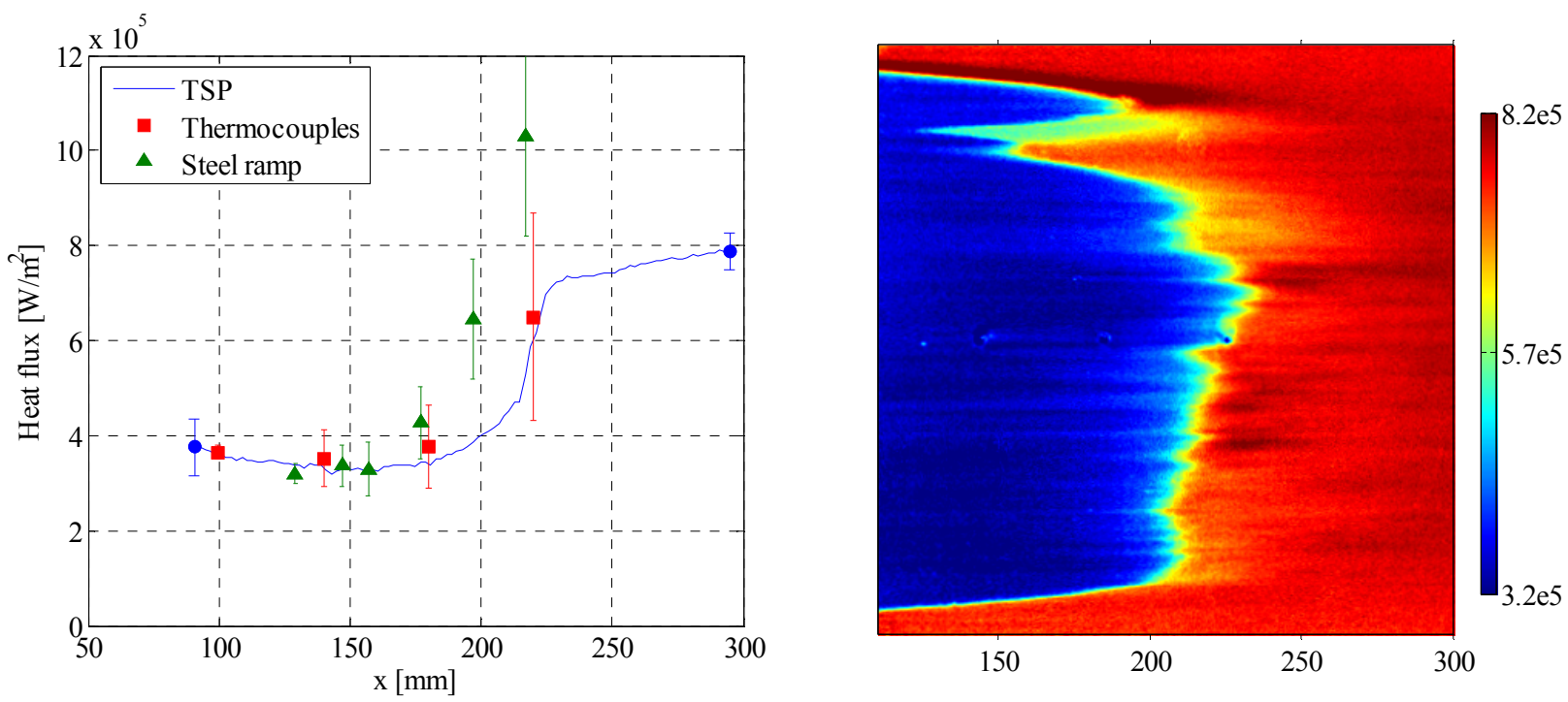

Figure 4: (Left) Heat-flux profile along the ramp center-line as measured by surface-mounted thermocouples and in-situ corrected TSP. Thermocouple measurements from an uncoated steel ramp at the same test conditions are also shown. (Right) Mean heat-flux distribution on the intake ramp recorded during the steady test time for the temperature distributions shown in figure 3 . The color scale is in units of $\mathrm{W} / \mathrm{m}^{2}$.

\section{Combustor Measurements}

\section{A. Diagnostic Techniques for Combustion Experiments}

For these measurements, the model was instrumented with a total of 63 pressure transducers, distributed over the inlet ramp, combustion chamber walls and thrust surfaces; it was also equipped with probes to measure the freestream Pitot and static pressures, and a reference heat-flux value. In the combustion chamber, a single row of pressure transducers was installed on each of the injector- and cowl-side walls, extending out into the thrust nozzle; in each case this was along the symmetry line between two injection port-holes. The transducer type employed on the injector-side wall was the Kulite XCEL-100, with a maximum pressure range of between 170 and $700 \mathrm{kPa}$ (the higher sensitivity transducers were used on in the expansion nozzle). On the cowl-side wall, GE NPP301 $700 \mathrm{kPa}$ transducers were installed. These provide a similar DC-response to the Kulite transducers and similar linearity over a limited range of temperatures, but at a much reduced cost. Thermocouples for the measurement of heat-transfer rates were also installed on both combustion chamber surfaces, but these will not be discussed further in this paper. 
Two types of high-speed visualization of the combustion chamber were performed simultaneously in these experiments. First, schlieren images were recorded to identify the important flow structures. The schlieren setup consisted of a conventional Z-fold arrangement; images were recorded using a Phantom v1210 high-speed digital camera. The resolution, frame rate, and exposure time were typically $1280 \mathrm{x} 80$ pixels, $110 \mathrm{kfps}$, and $1 \mu \mathrm{s}$, respectively. The light source employed was a Cavilux Smart $690 \mathrm{~nm}$ visualization laser; a pulse width of $10 \mathrm{~ns}$ allowed the flow structures to be effectively frozen in a given frame. A narrow band-pass filter allowed the removal of any extraneous light. The position of the model relative to the HEG test-section windows limited the schlieren visualization region to approximately $220 \mathrm{~mm}$ of the combustor.

Second, $\mathrm{OH}^{*}$ visualizations were captured to determine the approximate flame locations. The $\mathrm{OH}^{*}$ visualization apparatus consisted of a Shimadzu HPV-1 high-speed camera used in conjunction with a LaVision HS-IRO intensifier. Both the model combustor sidewalls and the HEG test section were fitted with quartz windows to allow transmittance of the relevant wavelength $(310 \mathrm{~nm})$, and a custom band-pass filter of $50 \mathrm{~nm}$ width centered at $310 \mathrm{~nm}$ was placed in front of the intensifier to block both other flame emissions and the considerable self-luminosity produced by the HEG facility. Images were recorded at a frame rate of $16 \mathrm{kfps}$, with an integration time for the IRO of $8 \mu \mathrm{s}$. The large aspect ratio of the combustor combined with the limited resolution of the Shimadzu camera, required a compromise in terms of field-of-view versus resolution across the combustor: the typical setting was such that the combustor height corresponded to approximately 15 pixels. As $\mathrm{OH}^{*}$ and schlieren images were recorded simultaneously, to avoid blocking the schlieren light path the $\mathrm{OH}^{*}$ visualization apparatus had to be mounted at an angle of approximately $12^{\circ}$ offset from normal to the flow direction.

\section{B. Flow Visualization}

A number of experiments were carried out with equivalence ratios ranging from 0.36 to 0.50 . The qualitative behavior as determined from the schlieren visualizations and pressure measurements can be summarized as follows. For the lowest equivalence ratio, no unsteady choking was observed during or prior to the test time. For equivalence ratios of 0.39 or higher, a shock train similar to those seen in ref. 8 was observed to develop, typically slightly before the beginning of the steady test time. This shock train initially propagated upstream, but during the test time would invariably find a quasi-steady position at which it remained until the conclusion of the test period, whereafter the increasing effective equivalence ratio (resulting from the falling reservoir pressure) would cause it to continue propagating upstream. This quasi-steady position showed a strong dependence on the equivalence ratio: for $\mathrm{ER}=0.50$, the leading shock in the shock train was more than halfway up the combustor, whereas for $\mathrm{ER}=0.39$, it was lodged towards the very rear of the combustor. For equivalence ratios in the range $0.38-0.39$, the choking shock was observed to develop during the flow start-up, but was washed downstream out of the combustor during the test time and did not appear again until after the test time had concluded. The remainder of this section will be devoted to illustrating and exploring this unsteady and quasi-steady behavior.

In figure 5 we present a sequence of simultaneous schlieren and $\mathrm{OH}^{*}$ images from an experiment with a calculated steady equivalence ratio of $\mathrm{ER}=0.50$. The visible extent of the schlieren images extends from $56 \mathrm{~mm}$ (slightly upstream of the injector) to approximately $280 \mathrm{~mm}$. The $\mathrm{OH}^{*}$ visualization apparatus was centered towards the rear half of the combustion chamber; some care must be exercised in interpreting the quantitative $\mathrm{OH}^{*}$ levels here, since the response of the HS-IRO exhibited some spatial dependency with decreasing sensitivity away from center. At $2.1 \mathrm{~ms}$, we see the expected shock pattern from the injection barrel shock reflecting down the duct; the turbulent structures in the hydrogen jet are also clearly visible. In the $\mathrm{OH}^{*}$ image, no notable features are present. At $2.4 \mathrm{~ms}$, the leading shock in the incipient shock train (indicated by the red arrow) becomes visible towards the very rear of the combustor in the schlieren image. The general level of the $\mathrm{OH}^{*}$ intensity has increased, but no obvious features associated with this shock are apparent. As in ref. 8, we interpret this as meaning that no large-scale separated regions are present, since the increased residence time in a recirculating flow will lead to much enhanced $\mathrm{OH}^{*}$ production. Thus, we again conclude that thermal choking is driving this unsteady development. The subsequent images from 2.7 to $5.2 \mathrm{~ms}$ show the motion of the shock train up the combustor, with the trailing shocks becoming clearly visible. We now see, however, that there is a strong $\mathrm{OH}^{*}$ feature on the cowl-side wall accompanying the leading shock in the train. The passage of the shock-train is thus intensifying the combustion, perhaps through boundary-layer separation, but most likely simply through the post-shock conditions, with elevated pressures and temperatures, and decreased flow velocity, being more favorable to ignition. Such $\mathrm{OH}^{*}$ features were not seen in the equivalent central-combustor images in ref. 8 (figure 11); the most likely reason for this is that, in the earlier experiments, the visualization apparatus was focused on the combustor side wall, which tended to accentuate features in the injector-side boundary layer and diminish them elsewhere. In contrast, here the apparatus was focused on the approximate mid-plane of the combustor. From 5.2 to $6.4 \mathrm{~ms}$, the shock-train motion has been 
$\mathrm{t}=2.1 \mathrm{~ms}$

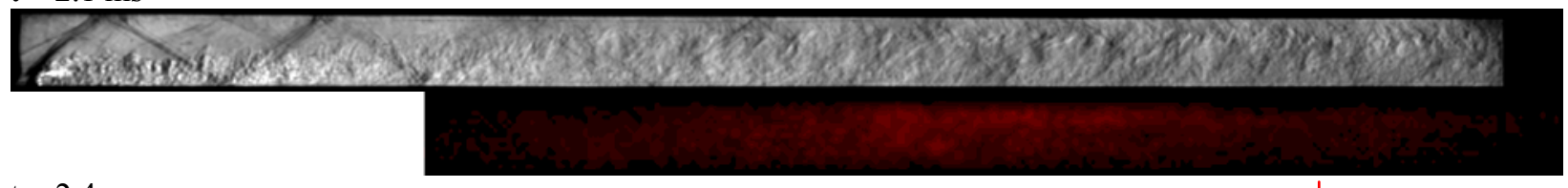

$\mathrm{t}=2.4 \mathrm{~ms}$

$+$

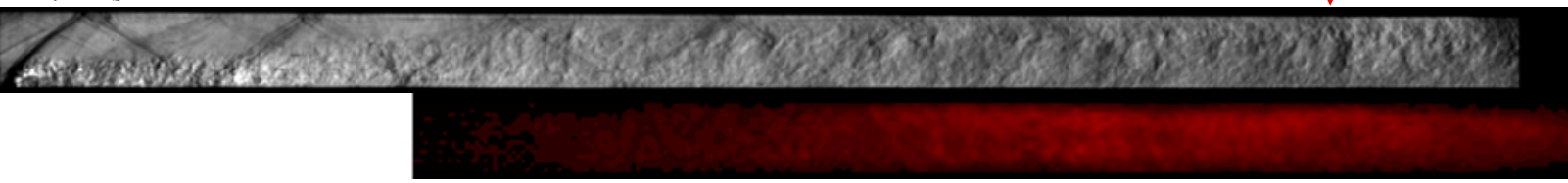

$\mathrm{t}=2.7 \mathrm{~ms}$

$+$

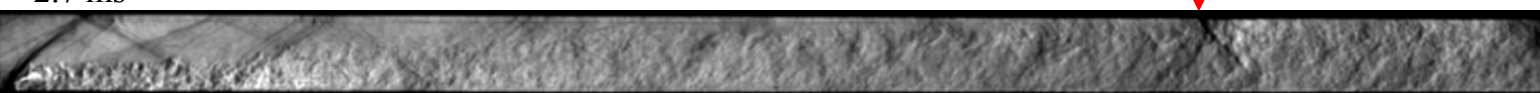

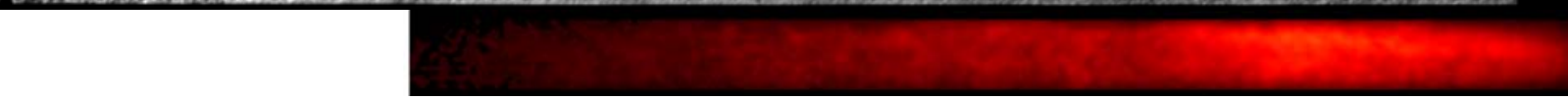

$\mathrm{t}=3.0 \mathrm{~ms}$

$\downarrow$

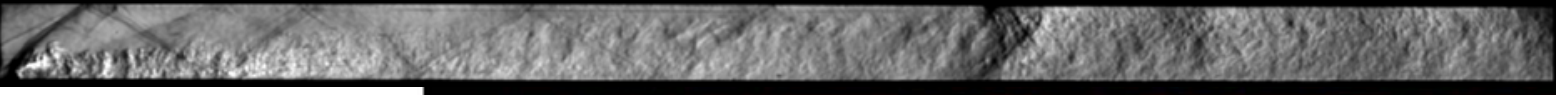
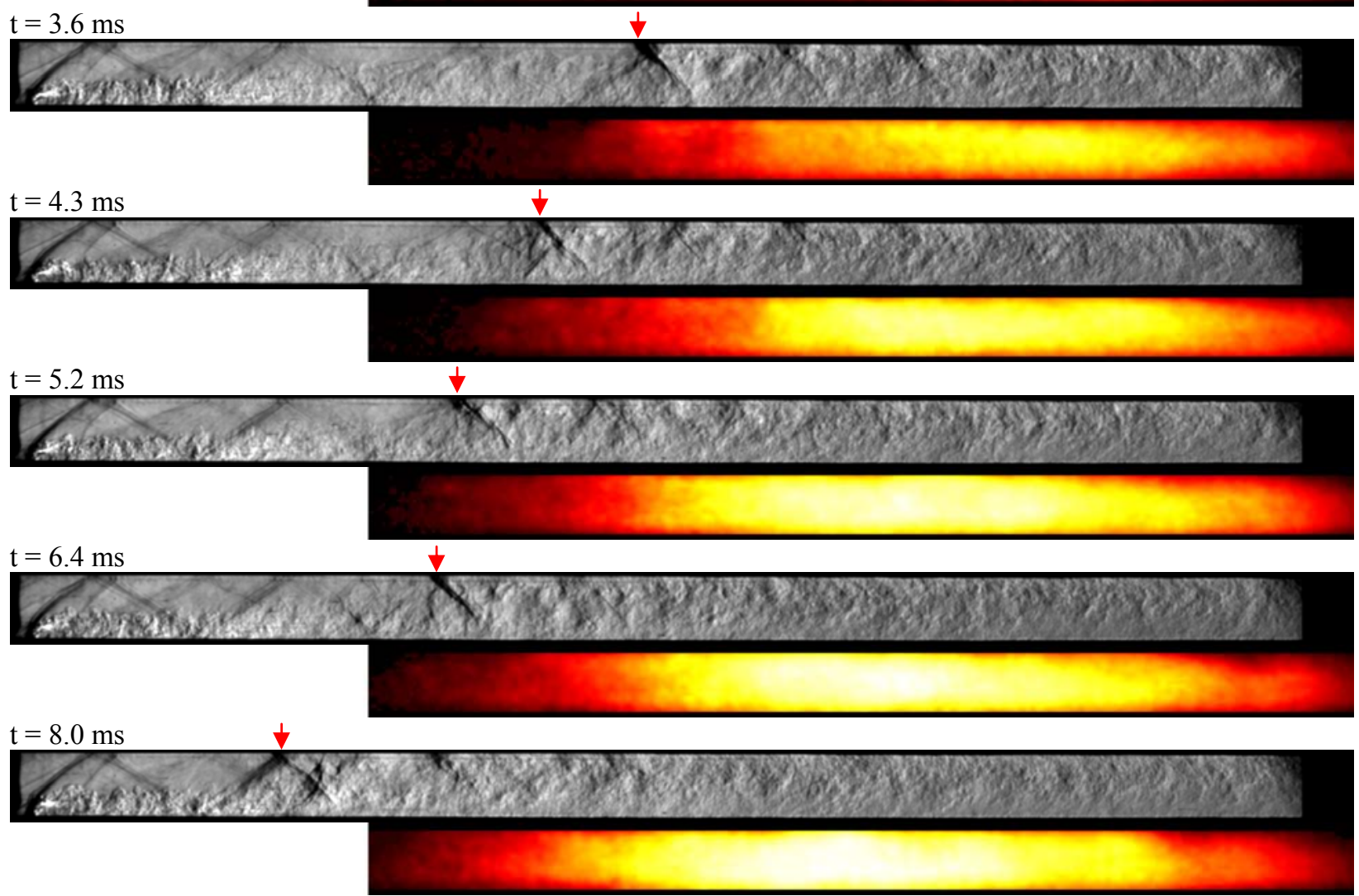

Figure 5: Simultaneous schlieren and $\mathrm{OH}^{*}$ chemiluminescence images showing the development of the combustor flow for $\mathrm{ER}=\mathbf{0 . 5 0}$

effectively terminated, and the leading shock is lodged on the cowl-side wall at a position corresponding roughly to the third reflection point of the injection shock. The upstream motion does not recommence until after the conclusion of the test time. 


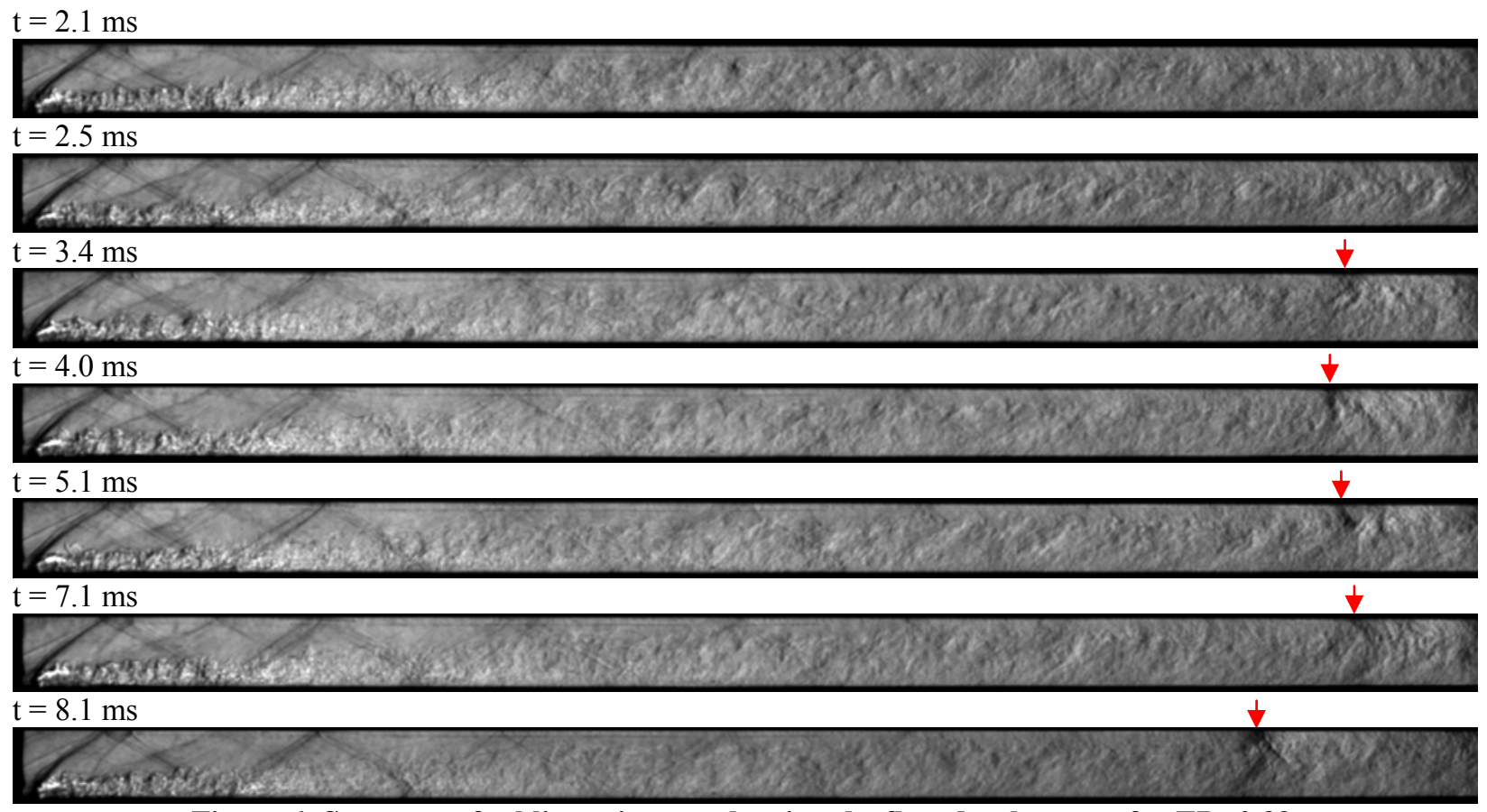

Figure 6: Sequence of schlieren images showing the flow development for $\mathrm{ER}=0.39$.

In figure 6, a schlieren sequence from an experiment with an equivalence ratio of 0.39 is shown. Here, the leading shock in the shock train is first visible at the rear of the combustor in the $3.4 \mathrm{~ms}$ image. It then moves only slightly forward before reaching a quasi-steady position at which it remains until $7.1 \mathrm{~ms}$. Again, no further flow development takes place until after the conclusion of the steady test time. The $\mathrm{OH}^{*}$ images for this development are not shown, but are as one might expect by comparing the schlieren shock train development with that in figure 5 .

\section{Analysis of Shock Motion}

The motion of the leading shock in the train on the cowl-side wall was tracked both in the schlieren images and using the relevant pressure measurements. To track the motion in the schlieren images, the streamwise gradient of the pixel intensity was calculated on a row of pixels close to the cowl-side wall, and the location of the maximum absolute gradient was assumed to correspond to the shock position. The passage of the shock train over the pressure transducers was identified by the steep rise in pressure signal. In figure 7, the shock motions determined using both of these methods are plotted in the form of x-t diagrams for a range of equivalence ratios from 0.38 to 0.50 . As described in the summary above, the general behavior is for the shock train to form slightly before the steady test time (at $\sim 3 \mathrm{~ms}$ ), propagate some distance upstream, and then find a quasi-steady location where it remains until the conclusion of the test time. The one exception to this description is the lowest equivalence ratio of 0.38 , for which the shock initially appears but is then washed downstream out of the rear of the combustor at approximately $5.7 \mathrm{~ms}$ and does not reappear until well after the conclusion of the test period. Generally, agreement between the schlieren and pressure results is very good. Occasionally a small discrepancy in positions is observed, typically with the schlieren-based measurement ahead of the pressure measurement, but this can be explained by the fact that the schlieren images are line-of-sight, whereas the pressure taps are located along a single streamwise line.

In figure 7, the influence of the equivalence ratio on the quasi-steady shock position is obvious, with larger equivalence ratios corresponding to positions further upstream. In figure 8, a quantitative estimate of the quasisteady position is made and plotted against the equivalence ratio. The shock position is clearly most sensitive to the exact value of the equivalence ratio at lower values, but as the equivalence ratio is increased, this sensitivity gradually decreases. The error bars here show the limits of the shock-train position during the quasi-steady period in each case (the selection of which was unavoidably slightly subjective). 


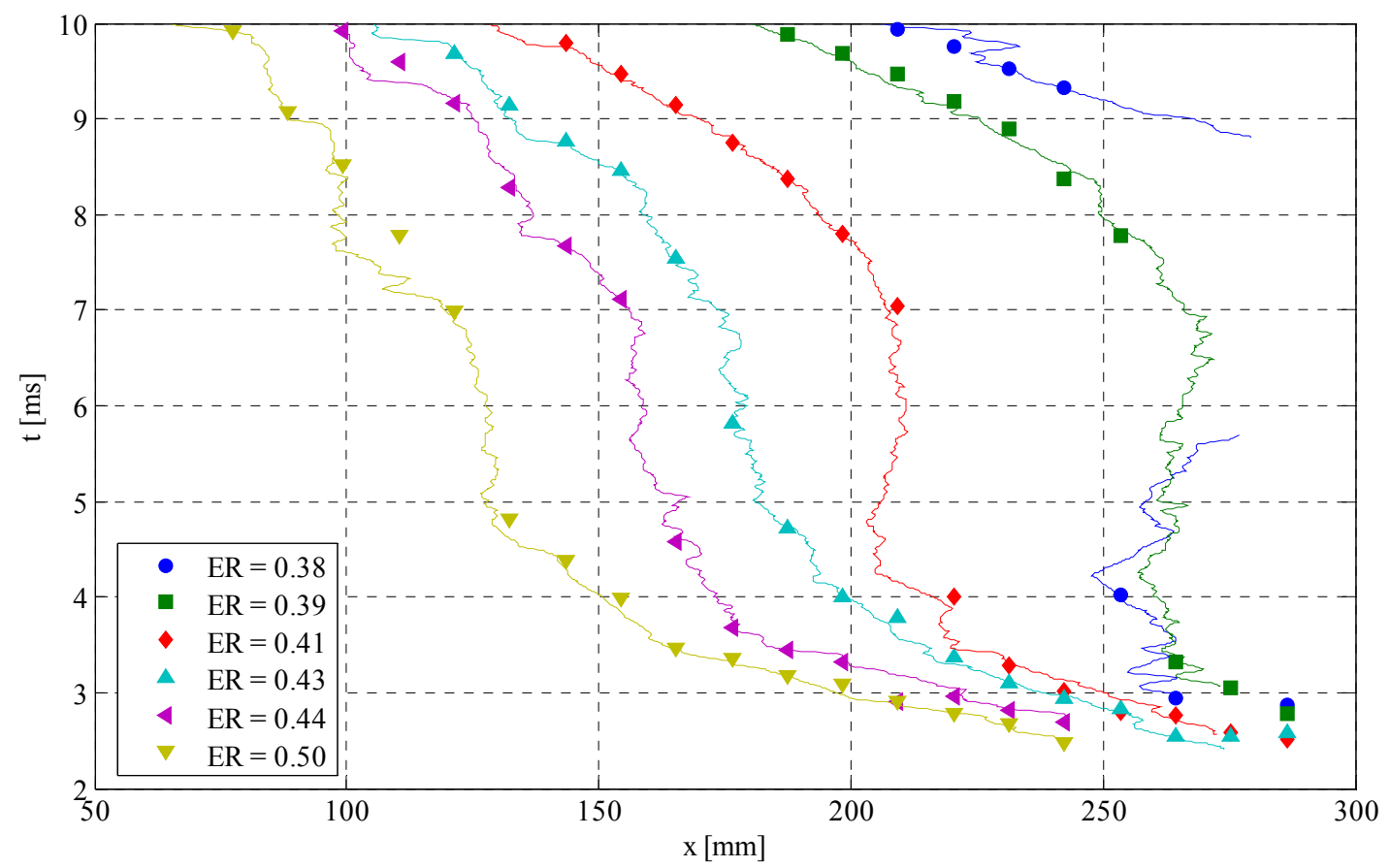

Figure 7: X-t diagrams showing the position of the leading edge of the shock train on the cowl-side wall for various equivalence ratios, as determined from schlieren sequences (lines) and pressure transducers (symbols)

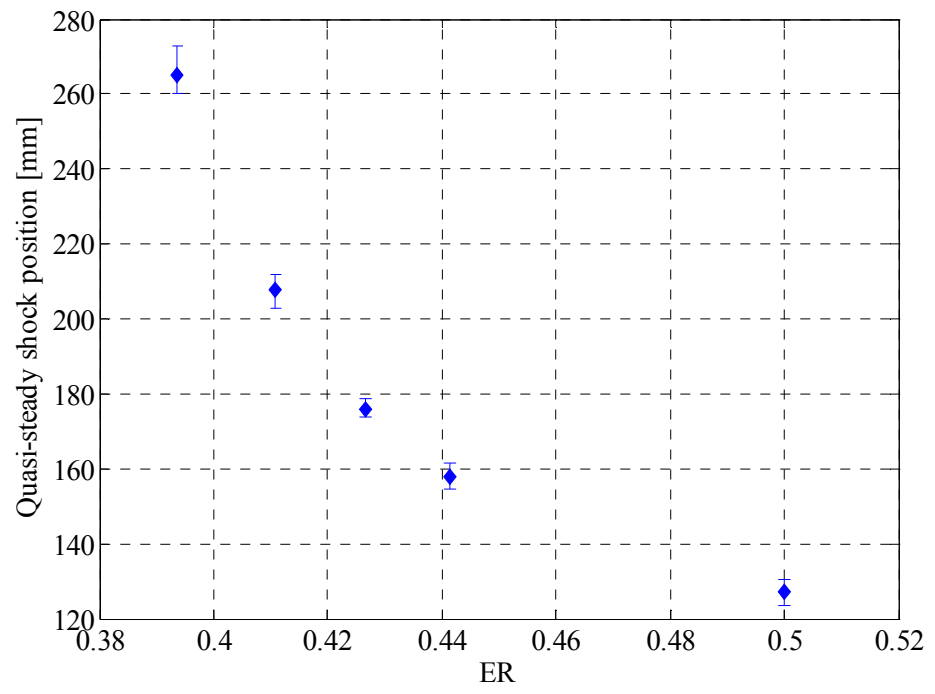

Figure 8: Quasi-steady position of leading shock on the cowl-side wall as a function of equivalence ratio

In figure 9 are plotted the pressure profiles on both the injector-side and cowl-side walls during the quasi-steady period in each experiment. For ER $=0.38$, a roughly steady increase in pressure is observed extending down both walls, with large-amplitude oscillations due to the reflecting injection shock superimposed on this trend. The general nature of this profile, however, is indicative of "clean" supersonic combustion. For larger equivalence ratios, the presence of the shock train is seen in the sharp pressure rise on both walls, which as expected, moves upstream as the equivalence ratio is increased. In all cases, the pressure rise on the injector-side wall is upstream of that on the cowl-side wall, despite the shock train appearing to be originating from the cowl-side wall in the schlieren images. The pressure oscillations caused by the trailing shocks in the train (seen most clearly for ER=0.41) are more apparent on the cowl-side wall, suggesting that the layer of injected hydrogen is dissipating the associated pressure rises. In the $\mathrm{ER}=0.50$ profiles, these oscillations have all but disappeared even on the cowl-side wall, indicating that 
some attenuation of the trailing structures is occurring (this can also be seen in the schlieren images of figure 5). In ref. 8, the onset of boundary-layer separation was suggested to be responsible for a breakdown in the shock-train structure, and this may also be the case here.
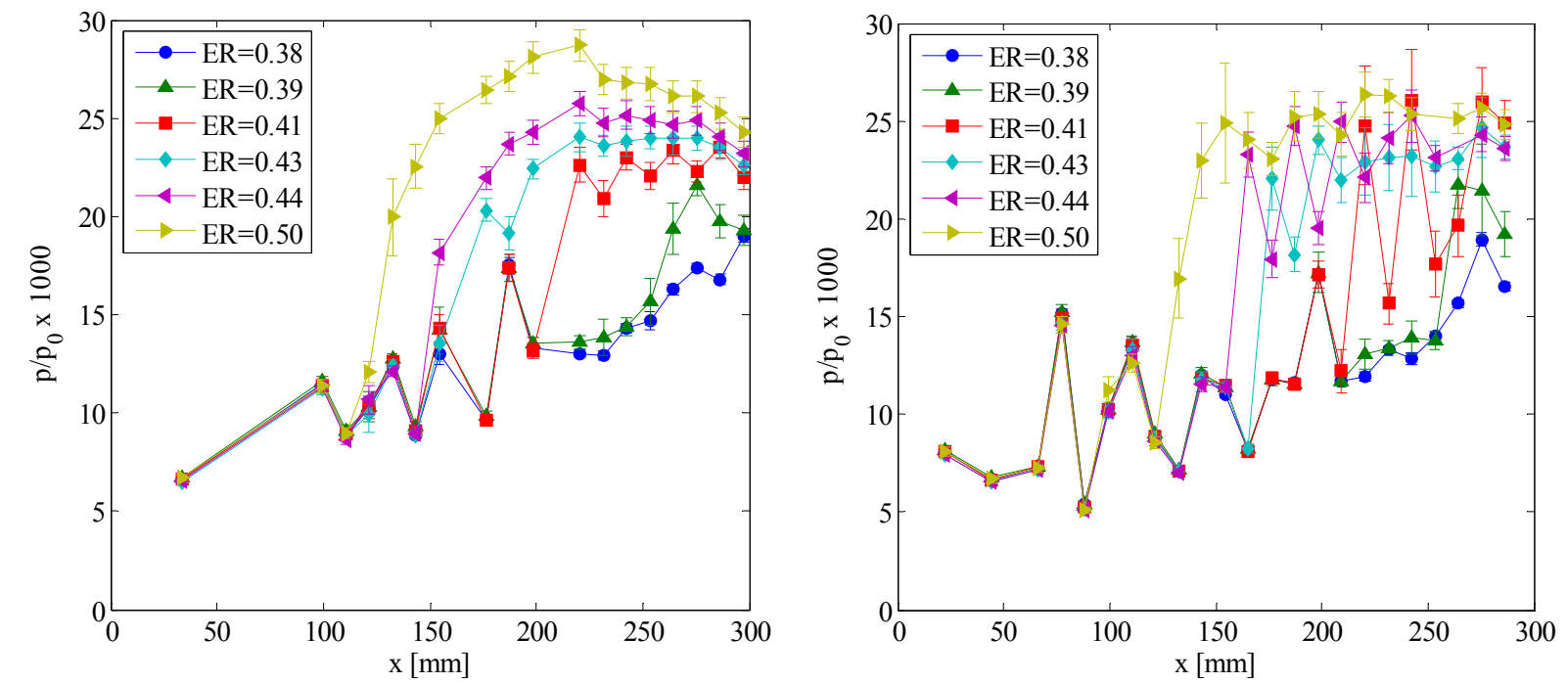

Figure 9: Mean static pressure profiles on the (left) injector-side wall and (right) cowl-side wall during the time period over which the shock train was stationary.

\section{Discussion}

In ref. 10, unsteady CFD simulations of the HyShot II combustor were performed to investigate further the choking behavior observed in the present experiments. As discussed in the introduction, localized thermal choking, whereby the heat release within the streamtube around the main combustion region causes the flow here to reach sonic conditions, was identified as the primary mechanism for this unsteady behavior. While the development of quasi-steady flow topologies with the shock train situated partway up the combustor was generally not observed in these CFD simulations, local thermal choking provides an explanation as to why such topologies can emerge. If the choking is restricted to an isolated streamtube, the flow can respond by sending pressure disturbances upstream that modify the flow topology and direct the heat release away from the choked regions. The formation of these new quasi-steady topologies is thus the flow's way of resolving the localized choking. For larger equivalence ratios, the streamwise location of the local thermal choking will move upstream; hence, the shock position will also need to move upstream of this location in order to be able to successfully modify the flow. The dependence of the quasisteady shock-train location on the equivalence ratio thus also follows readily from this explanation. If, in contrast, the heat release is such that the choking is global, i.e., sonic conditions are reached over the entire combustor cross section, no such resolution is possible: the combustor must respond by adjusting the inlet conditions via upstreampropagating pressure disturbances, leading to full unstart. For the present configuration, such behavior was observed for equivalence ratios of the order of $1.0^{8}$. Regarding the behavior observed in the present experiments, however, the question remains as to whether these are truly stable flow configurations or simply transient, quasi-steady ones. While the shock train motion appeared to have been effectively terminated in all of the cases studied here, the limited test time of the facility means that no solid conclusion can really be drawn.

One of the stated aims of this investigation was to identify the critical equivalence ratio corresponding to the onset of choking, which initially was also assumed would eventually lead to unstart. While the quasi-steady behavior found in the present experiments can clearly be characterized as a form of choking, it is not clear that it will in fact lead to unstart of the inlet. Nevertheless, the first appearance of the shock train gives a useful criterion, for example, in comparing to CFD simulations. As already described, for $\mathrm{ER}=0.38-0.39$ the shock appeared during the flow start-up period, but propagated downstream out of the combustion chamber during the steady test time; whereas, for $\mathrm{ER}=0.39$, the shock system remained in the combustor over the duration of the test time. Assuming no hysteresis in the formation and disappearance of the shock train then, it seems reasonable to conclude that the critical equivalence ratio lies in the range $0.38-0.39$ at the conditions studied. 
Finally, we address the apparent discrepancy between the present behavior and that observed at an equivalence ratio of approximately 0.5 in our previous investigation ${ }^{8}$. In that study, the choking phenomena for $E R=0.7$ were qualitatively similar to those observed here, with initial upstream shock-train propagation followed by the development of a quasi-steady flow topology. At the lower equivalence ratio, however, no pausing in the shock-train motion was observed. By comparing the time-developing reservoir pressure profiles in the two cases (e.g., that shown in figure 2), this apparent discrepancy is readily explainable. In the previous experiments, the test condition was slightly undertailored, with a continually decreasing reservoir pressure and thus, due to the constant injection pressure, an equivalence ratio that increased gradually during the test time and more rapidly after it. This meant that the initial shock-train development occurred on a longer time-scale, and also due to the shorter test time, the period of steady flow was insufficient for the development of the presently observed quasi-steady topologies.

\section{Conclusions}

A series of experiments has been carried out in the HEG wind tunnel, simulating the HyShot II scramjet configuration at $28-\mathrm{km}$ altitude conditions, with the primary aim of better understanding the combustor behavior close to the critical equivalence ratio at which the first onset of choking occurs. Measurements were made with fastresponse pressure transducers, and simultaneous high-speed schlieren $/ \mathrm{OH}^{*}$ chemiluminescence imaging was employed to visualize the interaction between the flow and combustion features during the choking development. For equivalence ratios larger than 0.39 , choking was associated with the formation of a shock train, which initially propagated up the duct, but thereafter halted, resulting in a new quasi-steady flow topology. The quasi-steady position depended strongly on the equivalence ratio, with higher ERs resulting in positions further upstream. This behavior was explained in terms of the "local" thermal choking observed computationally in ref. 10. For equivalence ratios in the range $0.38-0.39$, a choking shock initially developed, but was washed out of the combustor during the test time; for $\mathrm{ER}=0.36$, no evidence of a shock system was observed until after the steady test time. Thus, our estimate of the critical equivalence ratio (i.e., that corresponding to the first appearance of the choking shock) is in the range $0.38-0.39$.

\section{Acknowledgments}

We wish to acknowledge the HEG technical staff: Ingo Schwendtke, Mario Jünemann, Bartek Klaskala, Patrick Schenke, and Sarah Trost for assistance in preparing the model and carrying out the experimental runs in HEG, and Sebastian Karl for useful discussions.

\section{References}

${ }^{1}$ Heiser, W.H., and Pratt, D.T., "Hypersonic Airbreathing Propulsion”, AIAA Education Series, Washington, DC, 1994.

${ }^{2}$ Musielak, D., "Year in Review: High-Speed Air-Breathing Propulsion", Aerospace America, December 2011.

${ }^{3}$ Wieting, A. R., "Exploratory Study of Transient Unstart Phenomena in a Three-Dimensional Fixed-Geometry Scramjet Engine”, NASA TN D-8156, March 1976.

${ }^{4}$ Tan, H.-J., Guo, R.-W., "Experimental Study of the Unstable-Unstarted Condition of a Hypersonic Inlet at Mach 6", Journal of Propulsion and Power, Vol. 23, No. 4, 2007.

${ }^{5}$ Wagner, J.L., Yuceil, K.B., Valdivia, A., Clemens, N.T., Dolling D.S., "Experimental Investigation of Unstart in an Inlet/Isolator Model in Mach 5 Flow", AIAA Journal, Vol. 47, No. 6, 2009.

${ }^{6}$ Shimura T., Mitani, T., Sakuranaka, N., Izumikawa, M., "Load Oscillations Caused by Unstart of Hypersonic Wind Tunnels and Engines", Journal of Propulsion and Power, Vol. 14, No. 3, 1998.

${ }^{7}$ O’Byrne, S., Doolan, M., Olsen, S.R., Houwing, A.F.P., “Analysis of Transient Thermal Choking Processes in a Model Scramjet Engine", Journal of Propulsion and Power, Vol. 16, No. 5, 2000.

${ }^{8}$ Laurence, S., Martinez Schramm, J., Karl, S., and Hannemann, K., “An Experimental Investigation of Steady and Unsteady Combustion Phenomena in the HyShot II Combustor", AIAA Paper 2011-2310

${ }^{9}$ Paull, A., Alesi, H., Anderson, S., "The HyShot flight program and how it was developed," AIAA/AAAF 11th International Space Planes and Hypersonic Systems and Technologies Conference, Orleans, France, 2002.

${ }^{10}$ Karl, S., Laurence, S., Martinez Schramm, J., and Hannemann, K., "CFD Analysis on Unsteady Combustion Phenomena in the HyShot-II Scramjet configuration", $18^{\text {th }}$ AIAA International Space Planes and Hypersonic Systems and Technologies Conference, 2012 (submitted for publication).

${ }^{11}$ Hannemann, K., Schnieder, M., Reimann, B., Martinez Schramm, J., "The influence and delay of driver gas contamination in HEG", 21st AIAA Aerodynamic Measurement Technology and Ground Testing Conference, Denver, CO, 19-22 June, 2000

${ }^{12}$ Hannemann, K., "High Enthalpy Flows in the HEG Shock Tunnel: Experiment and Numerical Rebuilding", 41st AIAA Aerospace Sciences Meeting and Exhibit, 6-9 Jan, Reno, Nevada, 2003 
${ }^{13}$ Liu, T., Campbell, B.T., Sullivan, J.P., Lafferty, J., and Yanta, W., "Heat Transfer Measurements on a Waverider at Mach 10 using Fluorescent Paint", Journal of Thermophysics and Heat Transfer, Vol. 9, No. 4, 1995.

${ }^{14}$ Kurits, I, and Lewis, M., "Global Temperature-Sensitive Paint System for Heat Transfer Measurements in Long-Duration Hypersonic Flows", Journal of Thermophysics and Heat Transfer, Vol. 23, No. 2, 2009.

${ }^{15}$ Chou, A., Ward, C.A.C., Letterman, L.E., Luersen, R.P.K., Borg, M.P., and Schneider, S., "Transition Research with Temperature Sensitive Paints in the Boeing/AFOSR Mach-6 Quiet Tunnel", AIAA Paper 2011-3872.

${ }^{16}$ Cook, W.J., and Felderman, E.J., "Reduction of Data from Thin-Film Heat Transfer Gages: A Concise Technique", $A I A A$ Journal, Vol. 8, No. 7.

${ }^{17}$ Nagai, H., Ohmi, S., Asai, K., and Nakakita, K., "Effect of Temperature-Sensitive-Paint Thickness on Global Heat Transfer Measurement in Hypersonic Flow", Journal of Thermophysics and Heat Transfer, Vol. 22, No. 3, 2008. 